Pure \& Appl. Chem., Vo1.55, No.5, pp.819-832, 1983. Printed in Great Britain.
0033-4545/83/050819-14\$03.00/0

Pergamon Press Ltd。

(1983 IUPAC

POLYMER COMPOSITES OF RIGID AND FLEXIBLE MOLECULES

Motowo Takayanagi

Department of Applied Chemistry, Kyushu University, Fukuoka 812, Japan

\begin{abstract}
Aramid molecules are dispersed in the conventional polymers finely and uniformly in a state of molecules, microfibrils, fibrils or filaments by various methods such as coprecipitation from a common solvent. Very small fraction of rigid component as low as less than $5 \%$ shows an outstanding reinforcing effect on the matrix polymers, when the rigid component forms a quasi-3-dimensional lattice. Based on this model, the anisotropy of modulus was reasonably explained. Typical examples of molecular composites are the system of Aramid molecules or their block copolymers/aliphatic nylons, the system of Aramid molecules/NBR or PVC, and the system of surface-modified Kevlar fiber/Ionomer. A new type of self-reinforcing system is realized by using rigid molecules without liquid-crystal formation.
\end{abstract}

\title{
INTRODUCTION
}

Aramid fibers composed of rigid molecules such as poly(p-phenylene terephthalamide) (PPTA) represented by Kevler of du Pont Co. are employed as reinforcement of composite materials owing to their ultra high modulus/high strength properties in addition to their high heat endurance. Such high performance properties are ascribed to the rigidity of Aramid molecules, of which the fiber is composed. The new concept has been developed by the author that the basic principle providing the macro-fiber reinforced composite with excellent properties could be extended to the molecular level when the reinforcing molecules retain their rigidities and they are dispersed uniformly in ductile matrix polymers (Ref. 1). In actual case, the problem for realizing the molecular composite employing rigid molecules is in finding the method for dispersing rigid molecules finely and uniformly in the matrix, conquerring the strong intermolecular force of rigid molecules. It is not necessary to employ the spun filament, but the microfibrils of rigid molecules are enough for this purpose (Ref. $2 \& 3$ ).

These systems revealed that a very small amount of reinforcing molecules as low as less than $5 \%$ increased the fracture or yield stress by two to three times compared with those of matrix polymers and the transmission electronmicrograph indicated microfibrillar networks of rigid molecules.

When compared with the macro-fiber reinforced plastics, the molecular composites displayed the mechanical properties of almost the same level. It is noticeable that FRP employs the fiber reinforcements of as much as $40 \%$, while the molecular composites use only several percentages of rigid molecules. Networks of micro-fibrillar rigid molecules are effectively formed in the matrix and three-dimensional reinforcement is realized with simple process of coprecipitation with matrix polymers. Macro-fiber reinforced composites are always constructed at the beginning with fiber-winding or fiber-assembling, being followed by filling the inter-fiber space with ductile and adhesive resins. On the other hand, the molecular composites employing rigid molecules are processable with almost similar easiness to the matrix polymers. Thus, it may be said that a new type of raw materials has been born by adding a small amount of fine polymers with reinforcing functions. Microfibrillar network is not rigid but incomplete and thus, the composite is easily processable. In this meaning, a quasi-three-dimensional lattice model was proposed, which enables us to evaluate the anisotropy of modulus. The results will be shown for the cured composites of PPTA and NBR. A new type of proton substitution reaction of CONH in PPTA molecule was found by the author (Ref. 2). Unusual effectiveness of reinforcement was found for the system of the surfacemodified PPTA filament/sodium ion-type ionomer. Only addition of 2.2 vol\% of chopped strands of Kevlar fibers surface-modified with carboxymethyl group to ionomer resulted in remarkable increase of yield stress of ionomer by a factor of 3.5 at room temperature. This example belongs to the macro-fiber reinforcement, except a small amount of usage of filaments compared with the macro-fiber composites. The effectiveness is very close to that of the macrofiber composites. Actually, a quasi-3-dimensional model is again applicable to the modulus of this system. This example suggests that the fineness of reinforcement is not necessarily a sole factor but the way of interaction between matrix and reinforcement is also important. Various sides from scientific and engineering view points will be given for the above men- 
tioned systems, although the origin of the three-dimensionally developed network is opened for the future problem at present.

Recently, poly(p-phenylene terephthalate) modified with large fraction of p-acetoxy benzoic acid of ca. $60 \%$ was reported to show the characteristics of thermotropic liquid crystal (Ref. 4) and called "self-reinforcing plastics", which means that the reinforcing rigid segments of poly(p-oxybenzoic acid) are built-in within the molecules of the bulk polymers and give rise to orientation of molecules under stress, resulting in effective reinforcement. The molecular composite employing rigid molecules mentioned in this article is different from the above example in usage of very small amount of rigid component, being almost incapable of formation of liquid crystal. The reinforcing effect in molecular composite with rigid molecules is ascribed to its effective deposition in a net-work form in the polymer matrix. The common point in both cases is reinforcement achieved in molecular level. In this meaning, the molecular composite may be called another type of self-reinforcing plastics.

\section{PREPARATION AND CHARACTERIZATION OF MOLECULAR COMPOSITES}

The following examples of molecular composites are selected among other examples to show their characteristic methods of preparation and the effective reinforcement with rigid molecules. The results of characterization will be usefull to explain the origin of reinforcement.

\section{Aramid and nylon 6 or 66}

Preparation. The PPTA samples of various molecular weights were prepared by low temperature polycondensation of $\mathrm{p}$-phenylenediamine and terephthaloyl chloride in a mixed solvent of hexamethylphosphoramide (HMPA) and N-methyl-pyrrolidone (NMP) according to the method described in the literature (Ref. 5). Molecular weight of PPTA was controlled by the molar ratio of the reactants. The molecular weight was determined by measuring the intrinsic viscosity of PPTA in $97 \%$ sulfuric acid solution. Poly(p-benzamide) (PBA) was prepared by polycondensation of p-amino-benzoic acid by using triphenyl phosphate as a catalyst (Ref. 6). Sulfuric acid solution of PPTA or PBA and nylon 6 or 66 was extruded into a large amount of water. The precipitate was washed and the compound was heat-compressed at $240^{\circ} \mathrm{C}$ for nylon 6 and $290^{\circ} \mathrm{C}$ for nylon 66 .

Characterization. Figure 1 shows the electronmicrograph of surface replica of a fracture surface of the blend of PPTA/nylon 6 (7/93). PPTA of $\mathrm{M}_{\mathrm{V}}=12300$ was employed. The small holes of ca. $30 \mathrm{~nm}$ in diameter are uniformly dispersed and triangular shadow is always associated with each hole. This means that PPTA microfibrils are broken with their full strength under strong adherence at the micro-fibril surface. To clarify the dispersed state of microfibrils of PPTA in the composite, the matrix resin of nylon 6 in the composite was extracted with $99 \%$ formic acid and the remaining PPTA microfibrils were surveyed with electronmicroscope. Figure 2 shows that the PPTA microfibrils of $30 \mathrm{~nm}$ in diameter are continuously extending and branching to form a network. The bulk of molecular composite is composed of three-dimensionally developed PPTA microfibrillar networks and nylon 6 matrix fills up the major space of the composite. This network is not considered to be perfect, but rather incomplete by taking into account of the processability of the composite with heatcompression.

The strong interaction between PPTA fibril and nylon 6 matrix was found by the DSC curves, the x-ray diffraction, and the dilatometry. The DSC curves indicated the beginning of crystallization at $210^{\circ} \mathrm{C}$ for the composite, while $185^{\circ} \mathrm{C}$ for pure nylon 6 , when the melt was cooled down from $260^{\circ} \mathrm{C}$ with cooling rate of $10^{\circ} \mathrm{C} / \mathrm{min}$. Intensity curves of wide angle $\mathrm{x}-\mathrm{ray}$ diffraction of nylon 6 and the composite when quenched from the melt showed diffused curve and the $\alpha$ form crystal of nylon 6 , respectively. This means that nylon 6 crystallization is accelerated at the presence of PPTA fibrils. Similar evidence was obtained with isothermal crystallization curves. For example, Avrami's constant of 2 was obtained for the composite, while 4 was found for pure nylon 6 (Ref. 1). The similar characterization was conducted on the system of PBA and nylon 6, and almost the same results were obtained (Ref. 7). The strength of interfacial interaction between fibril surface and nylon 6 shows decreasing tendency with decreasing molecular weight. The well-developed surface of fibril composed of high molecular weight PPTA seems to be preferable for nucleation and overgrowth of nylon 6 . Further, to confirm the state of interfacial boundaries of PPTA and nylon 6, one denier filament of Kevlar filament was embedded in the matrix of nylon 6 , the system was once melted and isothermally crystallized at $210^{\circ} \mathrm{C}$ for $45 \mathrm{hrs}$. The polarization optical microscope under crossed nicols revealed that the interfacial zone of $\mathrm{ca}$. $3 \mu \mathrm{m}$ thickness exists close to the filament surface, and in the space between the interfacial zone and the spherulitic zone in the bulk of nylon 6, there exists the intermediate zone. The micro-beam x-ray diffraction with $10 \mu \mathrm{m}$ beam in diameter showed slightly different patterns between the interfacial and intermediate zones. Based on the diffractions of (200), (002) and (202) planes, it was concluded that, in the interfacial zone, the $a^{*}$-axis is oriented perpendicular to the filament surface with the $b-$ and the c-axis rotating around the $a^{*}$-axis, while in the intermediate zone, the a-axis is oriented perpendicular to the filament surface with the b- and the c-axis rotating around the a-axis. The latter type of orientation accords with the 


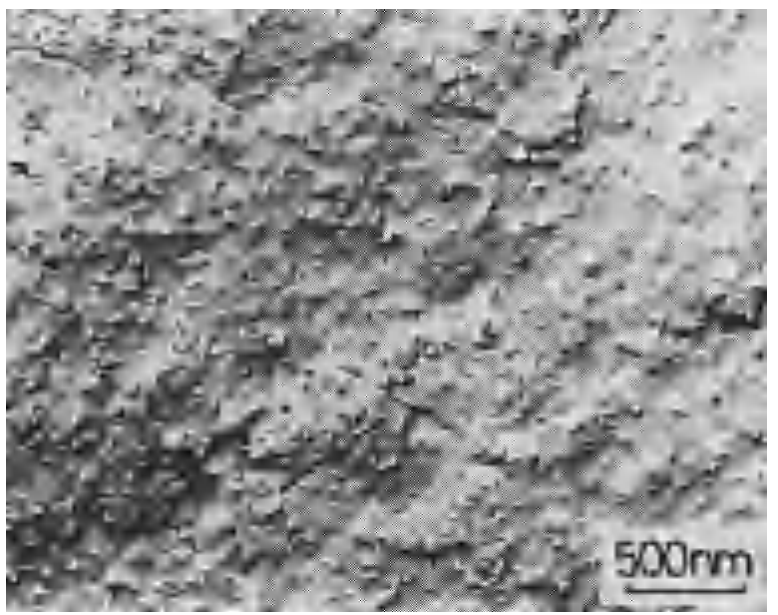

Fig. 1. Electronmicrograph of surface replica of a fractured surface of the blend of PPTA/nylon $6(7 / 93)$.

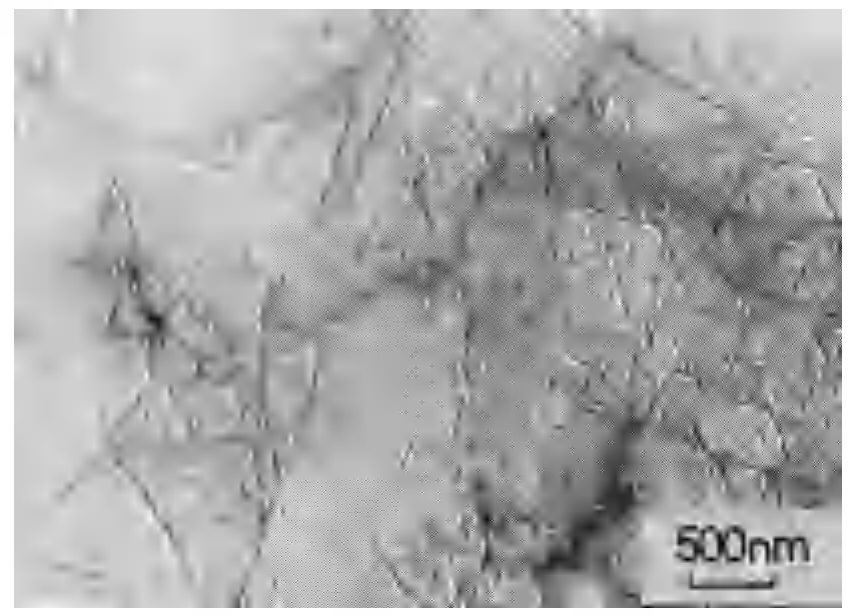

Fig. 2. Electronmicrograph of microfibrillar networks of PPTA, which remain after extraction of nylon 6 with formic acid from the molecular composite of PPTA/nylon $6(10 / 90)$.

lamellar structure of normal nylon 6 spherulite. The unusual orientation in the interfacial zone will be formed by the strong nucleating function of the filament surface (Ref. 8). The molecular composite composed of PPTA microfibrils and nylon 6 showed a statistically homogeneous birefringent image under polarization optical microscope with crossed nicols in the absence of spherulitic texture. However, more detailed inspection revealed that locally birefringent fibrous image was detected, especially when the high molecular weight PPTA was used as the component. Such fibrous images are supposed to be composed of row structure of nylon 6 overgrown on the surface of microfibril of PPTA with ca. 30nm in diameter. Microfibril itself was too small to detect with optical microscope.

Composites of block copolymers of Aramid and nylon 6 or 66

Preparation. To improve the dispersion of PPTA or PBA molecules into the nylon matrix, block copolymers of Aramid molecules with nylon block was tried to prepare. P-phenylene diamine and excess terephthaloy 1 chloride are reacted in HMPA-NMP-LiC1 solution. Chain ends of polycondensate are terminated with acid chloride chain ends. Such PPTA molecules were reacted with hexamethylene diamine and adipoyl chloride at the presence of triethylamine as an acid acceptor to give a PPTA-nylon 66 multi-block copolymer. The product was slightly 


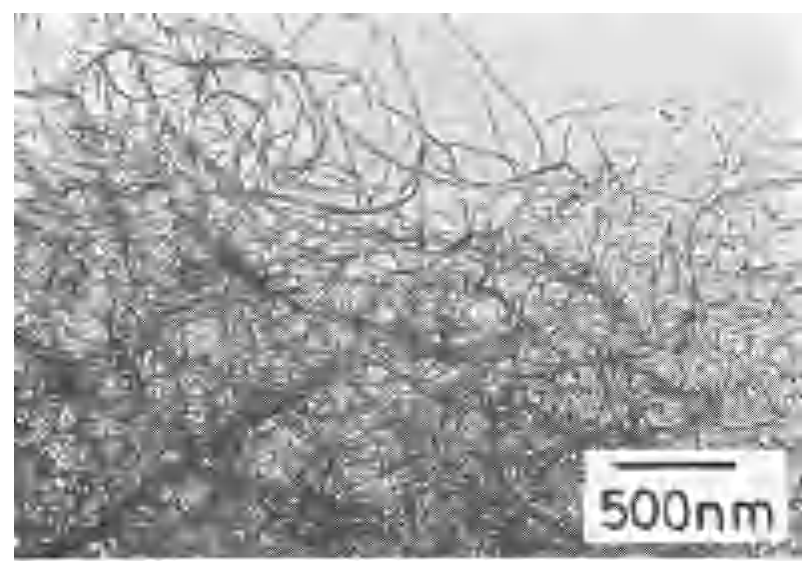

Fig. 3. Transmission electronmicrograph of PPTA microfibrils prepared by coagurating a sulfuric acid solution of PPTA in acetone under ultra-sonic irradiation.

soluble in formic acid and the molecular weight of PPTA at the first stage was 4200. PPTAb-nylon 6 copolymer was prepared as follows. Nylon 6 was reacted to obtain the acid chloride-terminated nylon 6 . Then, p-phenylenediamine was added to prepare PPTA block. The block copolymer was soluble in formic acid. PBA-b-nylon copolymer was prepared by the reaction of PBA and nylon 6 with triphenyl phosphite as a catalyst. The product was soluble in formic acid. The composites were prepared in a similar way as in the case of Aramid and nylon 6 or 66 .

Characterization. Birefringent images with polarization microscope with crossed nicols for the composite of Aramid-b-nylon copolymer and nylon were more homogeneous than that of Aramid and nylon system. There was no indication of fibrous image in the case of block copolymers. Dispersion of rigid segments into the matrix of nylon was performed more thoroughly. In a later part, the effect of flexible block attached to the hard segment of Aramid will be shown in the stress-strain behavior.

Preparation of PPTA microfibrils and their blends with poly (vinyl chloride) (PVC)

Preparation. A solution of $0.5 \mathrm{wt} \%$ PPTA $\left(\mathrm{M}_{\mathrm{V}}=36000\right)$ in sulfuric acid was sheared to form a thin layer on a glass plate and coagurated in acetone under ultrasonic irradiation. By decantation and ultracentrifuging, acetone was replaced by tetrahydrofuran. Figure 3 shows a transmission electronmicrograph of Pt-Pd shadowed microfibrillated PPTA. These microfibrils were flocculent in tetrahydrofuran (THF). PVC was dissolved THF, in which PPTA microfibrils are suspended. As-cast film of PVC including $2.4 \mathrm{wt} \%$ PPTA was turbid, suggesting inclusion of many voids. Once heat-compressed at $160^{\circ} \mathrm{C}$, the film became transparent. As-cast pure PVC film was quite transparent.

Characterization. Figure 4 shows a scanning electronmicrograph of the fractured surface of as-cast PVC-PPTA film. It is seen that the PPTA fibrils of ca. 10 $\mu \mathrm{m}$ in diameter are pulled out from PVC matrix. Interfacial adhesion between these fibrils and PVC matrix does not seem to be strong enough to bring about the fracture of fibrils. It is not clear that the microfibrils of ca. 10-30nm originally observed in Fig. 3 are playing the role of reinforcement. According to the Fig. 4, several microfibrils are supposed to form a fibril during the process of solvent-casting. For inspecting the interaction between PVC and PPTA, benzanilide was employed as the model compound of Aramid. The shifts of the amide A-band ( $\mathrm{N}-\mathrm{H}$ stretching) of $3320 \mathrm{~cm}^{-1}$ and the amide I-band ( $\mathrm{C}=0$ stretching) of $1655 \mathrm{~cm}^{-1}$ were observed for the composite of PVC and benzanilide. With increasing PVC content, the amide A-band shifted to the longer wave length of $3280 \mathrm{~cm}^{-1}$ and the amide I-band shifted to the shorter wave length of $1680 \mathrm{~cm}^{-1}$. This means that the hydrogen bond in benzanilide was dissociated by the presence of PVC. In accord with this change, the tan $\delta$ peak of PVC shifted to the lower temperature by blending benzanilide with PVC. These observation indicates that PVC molecules and PPTA surface shows a strong interaction when physical contact is sufficient. Actually, such strong interaction is realized when the temperature of the composite is increased above $\mathrm{Tg}$ of $\mathrm{PVC}\left(75^{\circ} \mathrm{C}\right)$ (Ref. 3). 


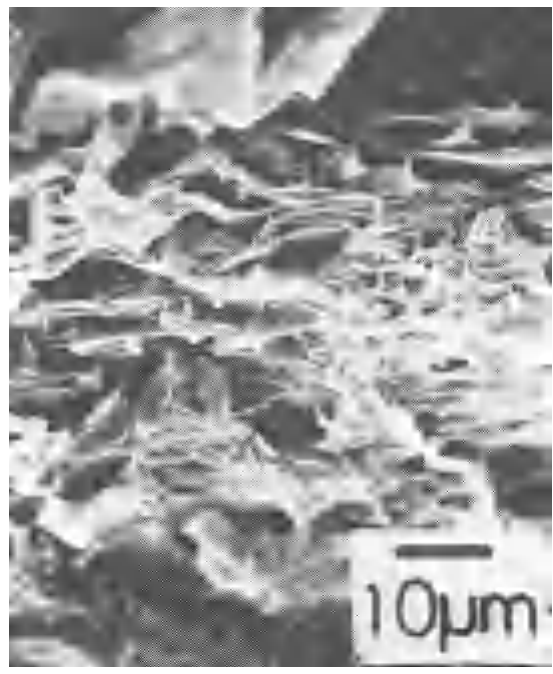

Fig. 4. Scanning electronmicrograph of fractured surface of a composite of PPTA microfibrils and PVC matrix prepared by casting the PVC solution in THF in which PPTA microfibrils are suspended.

Molecular composites of PPTA and nitrile-butadiene rubber (NBR)

Preparation. NBR is JSR-NBR N220S (Japan Synthetic Rubber Co.). Acrylonitrile content of NBR is $40 \mathrm{wt} \%$. PPTA was prepared by low temperature polycondensation as mentioned before. Intrinsic viscosity of PPTA in this case was $0.85,4.3$ and 5.0 , corresponding to the viscosity average molecular weight of 4900,21900 and 25100 , respectively. To dissolve PPTA and NBR raw rubber into a common solvent, PPTA was converted into metalated form, which allows PPTA molecules to dissolve into various kinds of polar solvents. First, powdered sodium hydride was dissolved in dimethyl sulfoxide (DMSO) and then PPTA was added. When a PPTA sample with low molecular weight is used, the mixture changes from the suspension of PPTA in DMSO to the transparent, deep red colored solution of metalated PPTA in DMSO after 4 hrs. When the high molecular weight sample is employed, the reaction system becomes a viscous jellylike mixture as the reaction proceeds. Additional DMSO is necessary to be poured into the reaction system to decrease the viscosity and make the sodium methylsulfinyl carbanion diffuse easily into PPTA particles. Reaction scheme is as follows (Ref. 2).

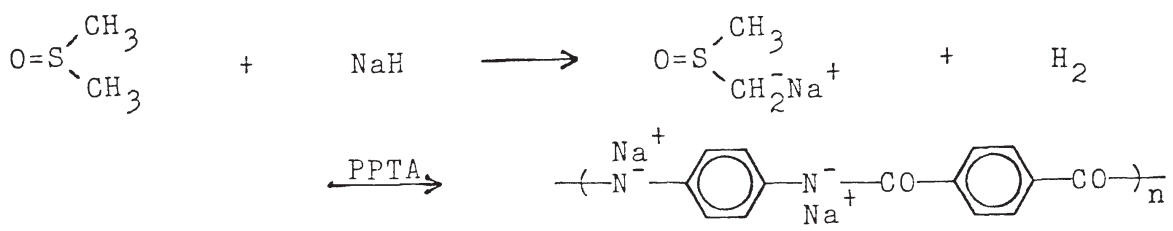

To N-metalated PPTA in DMSO, a solution of NBR in dimethyl formamide (DMF) is added. A red transparent solution is obtained. Blend polymer is precipitated by pouring its solution into a large amount of acidic water. With this process, metalated PPTA is regenerated and precipitated together with NBR molecules. The precipitate is washed with water to remove the organic solvent and the inorganic salt. The composite was dried at $50^{\circ} \mathrm{C}$ for $96 \mathrm{hrs}$ in vacuo.

The composite was milled on an open roll mill and compounded using the standard formulation listed in Table 1 . Rubber compound was cured at $150^{\circ} \mathrm{C}$ by compression molding for $60 \mathrm{~min}$. Curastometer indicated that the condition is optimum.

Characterization. Figure 5(a) shows the polarization optical micrograph under crossed nicols for the cured film of the polymer composite with PPTA/NBR $=10 / 100$ (by weight). The molecular composite shows an image of wholly birefringent colors in absence of discernible shape. For comparison, Fig. 5(b) shows the corresponding NBR vulcanizate including chopped strands of Kevlar filaments. To clarify the dispersed state of microfibrillar PPTA in NBR matrix, an uncured sample of the composite was extracted by DMF (the solvent of NBR) and the remaining image was inspected. As in the extracted molecular composite of nylon 6/PPTA with formic acid, microfibrils of PPTA could be detected like leaf veins. Diameter of elemental fibril was ca. 10-30nm, which is similar to that found in the nylon 6/PPTA composite.

In addition to the mechanical properties such as ultimate properties, temperature dependence of dynamic viscoelasticity, and tear strength, swelling behavior of NBR gum stock, black stock, and the corresponding PPTA composites with NBR ( $6.4 \%$ reinforcement) were inspected. The swollen shape of gum and black stocks in dichloromethane was spherical, but the shape of 
TABLE 1. Standard recipe for compound preparation

\begin{tabular}{lrlc}
\hline & Part by weight $(\mathrm{phr})$ & Part by weight (phr) \\
\hline NBR & 100.0 & Antioxidant* & 1.0 \\
Zinc oxide & 3.0 & Vulcanization & 0.7 \\
Sulfur & 1.5 & accelerator** & $2.5-10$ \\
Stearic acid & 1.0 & PPTA & \\
\hline
\end{tabular}

* IPPD (N-pheny1-N'-isopropy1 p-phenylene diamine)

** TBBS (N-tert-buty1-2-benzothiazol sulfenamide)

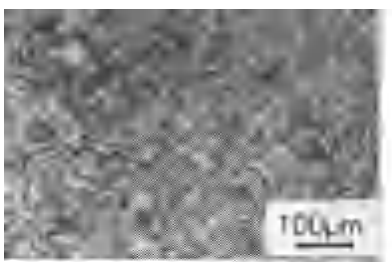

POLYMER COMPOSITE

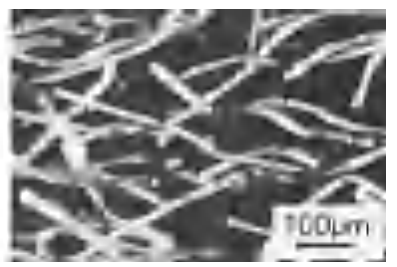

SHORT-FIBER COMPOSITE

PPTA $/$ NBR $=10 / 100$

(a)

(b)

Fig. 5. Polarization optical micrographs under crossed nicols for

(a) polymer composite of PPTA and NBR (PPTA/NBR $=10 / 100)$ and

(b) NBR including chopped strands of Kevlar filaments of 1 denier. Both samples are cured under the same conditions.

PPTA composite was ellipsoid with the shortened axis along the milling direction. It was noticed that the anisotropy of swollen sample of the PPTA composite is increased and the volume is decreased with increasing molecular weight of PPTA from 4900 to 25100.

Surface-modified Kevlar fiber and ionomer

Preparation. The ethylene type ionomer sample was Copolene (Asahi Dow Co., Ltd.), which contains methacrylic acid of $16 \mathrm{wt} \%$. The acid was ionized $35 \mathrm{~mol} \%$ by sodium ion. Starting material of poly(p-phenylene terephthalamide) was Kevlar 29. The PPTA fiber samples with the surface-modified by chemical reaction was obtained by the reaction of metalated PPTA surface with bromoacetic acid.

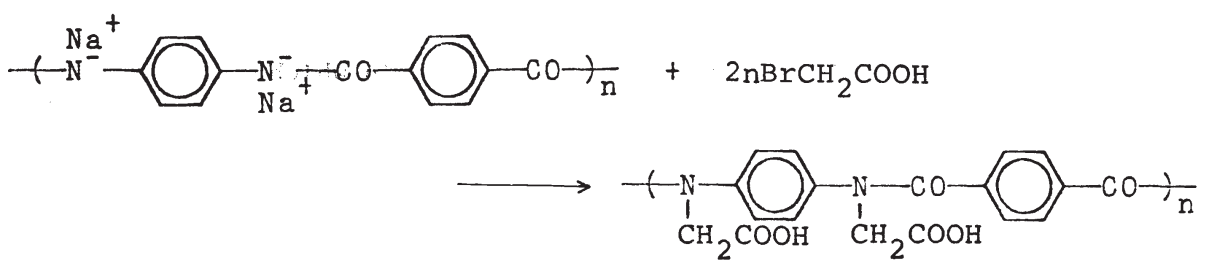

A suspension of the surface-modified PPTA fibers (0.34g) in xylene was added to a solution of ionomer $(9.66 \mathrm{~g})$ in a mixed solvent of xylene $(160 \mathrm{ml})$ and n-butyl alcohol $(40 \mathrm{~m} 1)$ at $120^{\circ} \mathrm{C}$. The composites were precipitated by pouring into a large amount of methyl alcohol, filtered and washed with methyl alcohol repeatedly. The composite was dried at $80^{\circ} \mathrm{C}$ for $10 \mathrm{hrs}$ in vacuo.

Characterization. Figure 6 shows the scanning electronmicrographs of PPTA filament (a), PPTA filament with the surface-modified by sodium ion (b), the cross section of metalated 

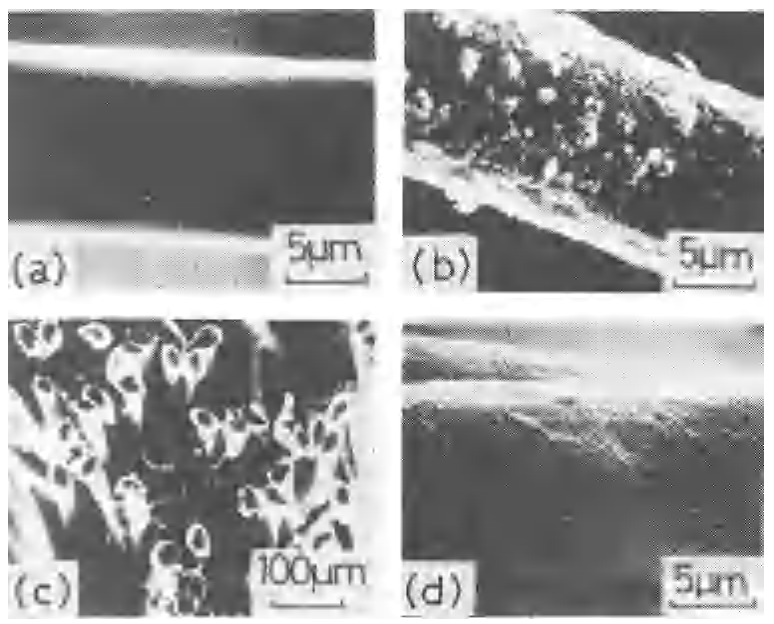

Fig. 6. Scanning electronmicrographs of (a) PPTA filament, (b) PPTA filament modified by sodium hydride, (c) the cross-sections of metalated PPTA filaments, and (d) PPTA filament surface-modified by carboxymethyl group.

fiber (filament assembly), and the PPTA filament with the surface modified by carboxymethyl group (d). The original PPTA fiber has a very smooth surface, whereas those of modified ones show much rougher surfaces. Figure $6(\mathrm{c})$ shows that the metalation reaction proceeded into the inner part of the PPTA fiber with the surface layer about $1 \mu \mathrm{m}$ thick. It is noticed that in the case of carboxymethyl-substituted sample (Fig. 6(d)), several little smooth portions are found, suggesting the presence of unreacted parts. Major part of surface layer was dissolved with sulfuric acid together with some parts of PPTA molecules. Infrared spectra of recovered portion showed distinctive absorption of carboxymethyl group at $1750 \mathrm{~cm}^{-1}$. Observation of fractured surface of the composite prepared by thermal compression showed indications of good fiber/matrix adhesion for both cases of surface-modified and unmodified samples. The difference was found in the fractured surface obtained at $77 \mathrm{~K}$, at which temperature the unmodified fiber was fractured at the interface, whereas the modified one showed still good indication of adhesion (Ref. 9).

\section{DYNAMIC TENSILE MODULUS AND LATTICE MODEL APPROACH}

One of the important mechanical properties is tensile modulus of the material. In the molecular composites, microfibrillar strands of rigid molecules are dispersed in the matrix of conventional polymers of low modulus, except in the case of the surface-decorated Kevlar system. Aramid molecules give a ultra-high modulus/strength filaments, as represented by Kevlar fiber. The highest modulus ever evaluated by us amounted at $88 \mathrm{GPa}$ at $123 \mathrm{~K}$ and $60 \mathrm{GPa}$ at $573 \mathrm{~K}$ (Ref. 10). Depending on the preparation method and molecular weight, the modulus of $150 \mathrm{GPa}$ is conceivable. To evaluate the modulus value of PPTA microfibrils separated from the dissolved state in a common solvent of PPTA and conventional polymers is almost impossible. By referring to the modulus value of Kevlar filament, calculation of the modulus of the composites were tried and the modulus value of microfibrillar PPTA was taken as an adjustable parameter.

Model for calculating the modulus of the composite was constructed by taking into account of the results of morphological observation. For example, Fig. 1 and 2 provide us with the image of model like a 3-dimensional microfibrillar lattice, in which microfibrils form strands of a network. The major space of the composite is filled-up with the matrix polymer. In the case of the composite of PPTA/NBR, it is necessary to evaluate the modulus anisotropy associated with the milling direction, which is realized by the modified lattice.

Aramid and nylon 6 or 66

Thermomechanical data. Figure 7 shows the dynamic tensile storage modulus, E' at $110 \mathrm{~Hz}$ as a function of temperature. Chain line in the upper figure is for the molecular composite including PPTA microfibrils of 4.1 vol\% with molecular weight of 12300 (curve 2), and chain line in the lower figure is for the composite of 4.1 vol\% PPTA with molecular weight of 980. For reference, the curve of nylon 6 is shown by dotted line (curve 3). Detailed discussion of relaxation processes was given in Ref. 1. Increased modulus and heat endurance of the composite compared with nylon 6 are easily seen. The composites were prepared by quenching the thermally compressed sheet at the temperature of molten nylon. 


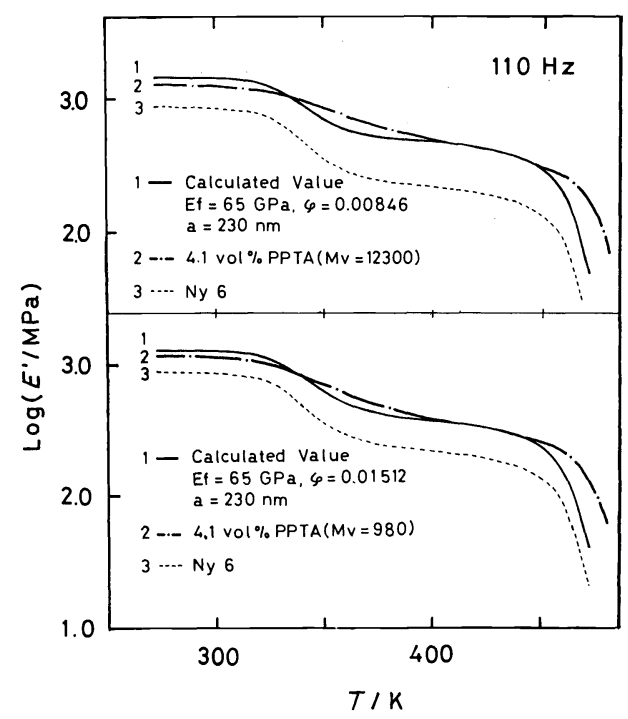

Fig. 7. $E^{\prime}$ vs temperature at $110 \mathrm{~Hz}$ for the observed (curve 2) and calculated (curve 1) moduli of the molecular composites and for nylon 6 (curve 3) received with the same process as the composites. Upper figure is for the composite including PPTA of $\mathrm{M}_{\mathrm{V}}=12300$, and lower figure is for the composite including PPTA of $\mathrm{M}_{\mathrm{V}}=980$.

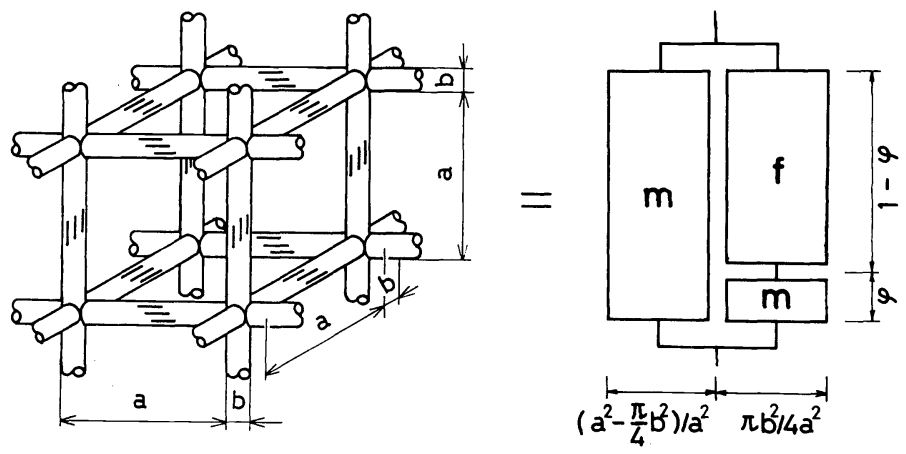

Fig. 8. Quasi-3-dimensional microfibrillar lattice model (left-side) and the equivalent mechanical model for modulus calculation (right-side).

" $a$ " denotes the distance between the neighbouring network strand and " $b$ " the diameter of the microfibrillar strand. " $m$ " denotes the contribution from the matrix and " $f$ " denotes the modulus contribution from the microfibril. " $\phi$ " represents the fraction of matrix connected to the network strand in series.

Evaluation of modulus based on the lattice model. First an ideal 3-dimensional microfibrillar lattice was constructed. Each strand has an diameter of "b" and strands forming cubic lattice have edge length of "a". Each strand extends along the three axes of Cartesian coordinate and the force is assumed to be supported by only the strands directing along the force. The connecting points has no sence of cross-link point, because at a very small strain level the distortion at that point is unnecessary to be taken into account.

In conclusion, to explain the modulus of the composite by using the reasonable modulus value of Aramid was impossible, giving higher modulus value in the high temperature region owing to the retention of high modulus through a whole temperature range. Thus, each strand is assumed to be incomplete to support the full force to the highest temperature, but it includes a small fraction of " $\phi$ " of the matrix, which induces the softening of the composites to meet with the actual behavior in such a high temperature region. The most acceptable model is constructed as shown in Fig. 8, which is called hereafter "quasi-3-dimensional 
microfibrillar lattice model". The diameter of fibril "b" was assumed to be $30 \mathrm{~nm}$, which accords with the result of observation in Fig. 1 with electron microscope. The distance between neighbouring strands "a" should be 100-200nm from the same micrograph. For the calculation of the modulus of the composite, it was assumed that the transversal modulus of microfibrils is comparable to that of the matrix and the total fraction of matrix is very large compared with the Aramid fraction, that is, "a" is enough larger than "b". Denoting " $\mathrm{E}_{\mathrm{f}}$ " as filament modulus of Aramid and " $\mathrm{E}_{\mathrm{m}}$ " matrix modulus, the composite modulus "E" and the volume fraction of Aramid " $\mathrm{V}_{\mathrm{f}}$ " are given by the following equation.

$$
\begin{aligned}
& E=\frac{a^{2}-\frac{\pi}{4} b^{2}}{a^{2}} E_{m}+\frac{\pi b^{2}}{4 a^{2}}\left(\frac{1-\phi}{E_{f}}+\frac{\phi}{E_{m}}\right)^{-1} \\
& v_{f}=\left(\frac{3}{4} \pi b^{2}-\frac{\pi}{2} b^{2}\right) / a^{3}
\end{aligned}
$$

The agreement between the calculated and the observed values is not necessarily satisfactory. The introduction of small amount of matrix component into the network strand is very sensitive and an appropriate evaluation of modulus of this component is also questionable.

The similar agreement was found for the systems of 8.2 vol\% and 4.0 vol\% of PBA dispersed in the matrix of nylon 6. Molecular weight of PBA was 8400 . Table 2 summarizes the values of parameters employed for calculation of moduli for the systems cited here.

TABLE 2. Parameter values for evaluating the moduli of the composites of Aramid and nylon 6

\begin{tabular}{llccc}
\hline Composites & $\mathrm{a}(\mathrm{nm})$ & $\mathrm{b}(\mathrm{nm})$ & $\mathrm{E}_{\mathrm{f}}(\mathrm{GPa})$ & $\phi$ \\
\hline 4.1 vo1\% PPTA $\left(\mathrm{M}_{\mathrm{v}}=980\right)$ & 230 & 30 & 23 & 0.0084 \\
4.1 vo1\% PPTA $\left(\mathrm{M}_{\mathrm{v}}=12300\right)$ & 230 & 30 & 65 & 0.01 \\
4.0 vo1\% PBA & 230 & 30 & 106 & 0.04 \\
8.2 vo1\% PBA & 160 & 30 & 106 & 0.04 \\
\hline
\end{tabular}

The value of " $b$ " was evaluated as $30 \mathrm{~nm}$ from the direct observation. The other parameters were adjusted to give the good agreement with the experimental data. The values of "a" are not so bad, since the microfibril is located at the distance of ca. 100-200nm with each other in the electronmicrograph. The Aramid modulus, $\mathrm{E}_{\mathrm{f}}$ seems to be reasonable. In the case of PPTA, the microfibrils are separated from the matrix when quenched, and no heat treatment was applied except in the process of thermal compression. Increase of molecular weight from 980 to 12300 gives rise to the increase of $\mathrm{E}_{\mathrm{f}}$ from 23 to $65 \mathrm{GPa}$. The structure of microfibril of PPTA becomes more well-developed in the latter case, giving higher modulus. The molecular composites employing PBA was prepared by annealing the system at $212^{\circ} \mathrm{C}$ for $48 \mathrm{hrs}$, which induces some increase of modulus, reflecting the effect of heat-treatment. Well-developed row structure of nylon 6 around the PBA microfibril might be preferable for increasing modulus. The value is higher than that of PRD 49 by an amount of $50 \%$.

Modulus anisotropy for the composites of PPTA/NBR and its evaluation

Figure 9 shows thermomechanical data for the composites of PPTA/NBR. Figure 9 (a) is for the composite employing PPTA of $\mathrm{M}_{\mathrm{V}}=4900$, (b) $\mathrm{M}_{\mathrm{v}}=21900$ and (c) $\mathrm{M}_{\mathrm{V}}=25100$. The experimental data are represented by the broken lines. With increasing molecular weight of PPTA, the anisotropy of the moduli is increased. The milling process before final curing process has an influence for orienting the microfibrils. The dotted curve of NBR gum stock is employed to calculate the modulus of the composite, since the matrix of the composite is supposed to be very close to the state of NBR gum stock. The microfibrils may be preferably oriented along the milling direction and reduced along the transverse direction in their fraction. Thus, the model is constructed to give such an anisotropy as indicated in each figure. The modulus along the milling direction is denoted by $\mathrm{E}_{11}$ and the network strands denoted by "a" respond to this, and the modulus perpendicular to the milling direction is denoted by "E1", to which the strands of "b" indicated in the figure respond. The fraction of the strand parallel to the thickness direction is supposingly very small and the strand "c" is assigned along this direction. Unfortunately the modulus along the thickness has not yet been evaluated. Anisotropy of the structure is reflected to the ratio of the axis length of $a: b$ : c. With increasing modulus anisotropy, the axis length varies distinctly in the order of 


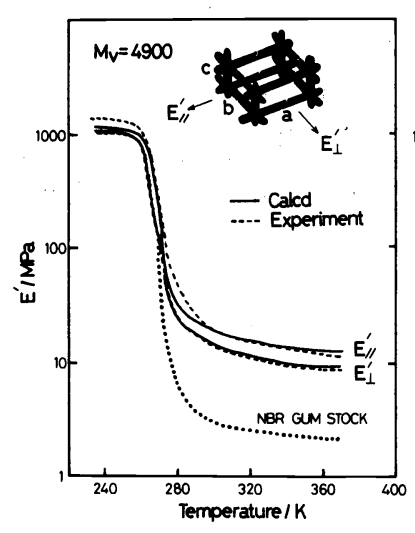

(a)

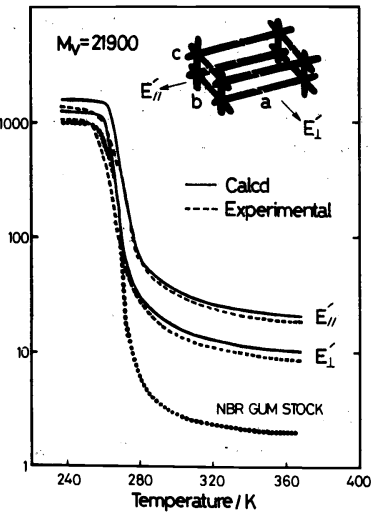

(b)

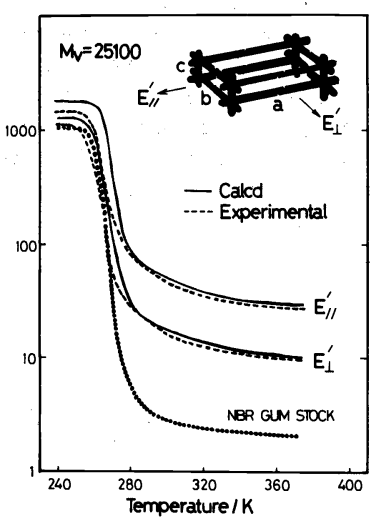

(c)

Fig. 9. $E^{\prime}$ vs temperature at $11 \mathrm{~Hz}$ for the cured composites of PPTA and NBR. The broken lines denotes the experimental data and the full lines are the calculated curves based on the quasi-3-dimensional lattice model as indicated in the figure. The curve of NBR gum stock is the data employed for calculation. The other parameters are listed in Table 3. Molecular weight of PPTA is 4900 for (a), 21900 for (b), and 25100 for (c). E 11 is measured along the milling direction and $E_{1}$ perpendicular to the milling direction.

$\mathrm{a}, \mathrm{b}$ and $\mathrm{c}$. Based on the observed diameter of microfibril, 30nm, the other parameters are evaluated to meet with the experimental data. Table 3 summarizes the results of evaluation for three kinds of the composites differing in molecular weight of PPTA employed for the composites.

It should be noticed that the value of $\mathrm{E}_{\mathrm{f}}$ is extraordinarily low, compared with the modulus value of Kevlar fiber of 70-130GPa. The value of $\mathrm{E}_{\mathrm{f}}$ in this case is rather understood as representing the net modulus for the fibril including defective zones generated by metalation reaction. In the previous case of the composites of Aramid/nylon, such effects were taken into account by introducing the fraction $\phi$ of the contribution from the matrix to the network strand. Here $\phi$ was estimated to be 0.0025 .

TABLE 3. Parameters of 3-dimensional model for the cured molecular composites of NBR and PPTA $\left(V_{f}=0.060\right)$. Microfibril diameter is assigned to $30 \mathrm{~nm}$.

\begin{tabular}{cccccc}
\hline Molecular weight of PPTA & a (nm) & b (nm) & c (nm) & $E_{\mathrm{f}}(\mathrm{GPa})$ & $\phi$ \\
\hline 4900 & 299 & 214 & 107 & 5 & 0.0025 \\
21900 & 407 & 195 & 97 & 18 & 0.0025 \\
25100 & 520 & 184 & 92 & 20 & 0.0025 \\
\hline
\end{tabular}

Evaluation of modulus for the composite of the surface-modified PPTA filaments/ionomer Figure 10 shows the experimental data of $E^{\prime}$ vs temperature for the composite of the surfacemodified PPTA with carboxymethyl group and ionomer. The curve of ionomer itself is also shown, which was employed for calculation of the modulus of the composite. 'The E' curve for the composite shows a remarkable effect in reinforcing the matrix of ionomer. The reinforcing effect is superior over the other ultra-high modulus fiber such as carbon fiber etc. The strong interaction at the interface between the modified surface of PPTA filament and ionic domains in ionomer is a governing factor to achieve excellent reinforcement. Heatendurance is raised by an amount of $60^{\circ} \mathrm{C}$ compared with ionomer.

The sheet of the composite was formed by thermal compression and the filaments are oriented biaxially parallel to the plane surface. Thus, the model is constructed for the random-inplane orientation as shown in the same figure. Here the anisotropy is taken into account between the thickness direction and the direction parallel to the sheet surface.

The agreement of the calculated modulus with the experimental data was obtained when the diameter of the filament is taken as $12 \mu \mathrm{m}$ from the SEM observation, $\mathrm{V}_{\mathrm{f}}$ is 2.2 vol\% of actual data, $E_{f}$ is assumed to be $40 \mathrm{GPa}$ with $\mathrm{a}=323 \mu \mathrm{m}$ and $\mathrm{b}=\mathrm{a} / 10=32 \mu \mathrm{m}$. The selection of parameter, $\phi$ is very sensitive as seen in Fig. 10. Curves 1 and 2 are the original data. Curves $3,4,5$, and 6 are drawn by assuming $\phi=0,5 \times 10^{-5}, 1 \times 10^{-4}$, and 0.01 , 
respectively. Curves 4 and 5 show fairly well agreement with the composite data, while curve 6 is too low to reproduce the modulus curve of the composite. Curve 7 is calculated by using the modified Halpin-Tsai equation (Ref. 11).

It is a very interesting result that macro-filament of $12 \mu \mathrm{m}$ in diameter also shows a remarkable reinforcing effect by using only a very small amount of filaments of as low as 2.2 vol\%. The reason is not clear, but it seems to be very important that the filament forms a quasi3-dimensional network. The strong aggregation tendency of Aramid fibrils and fibers might play such a role.

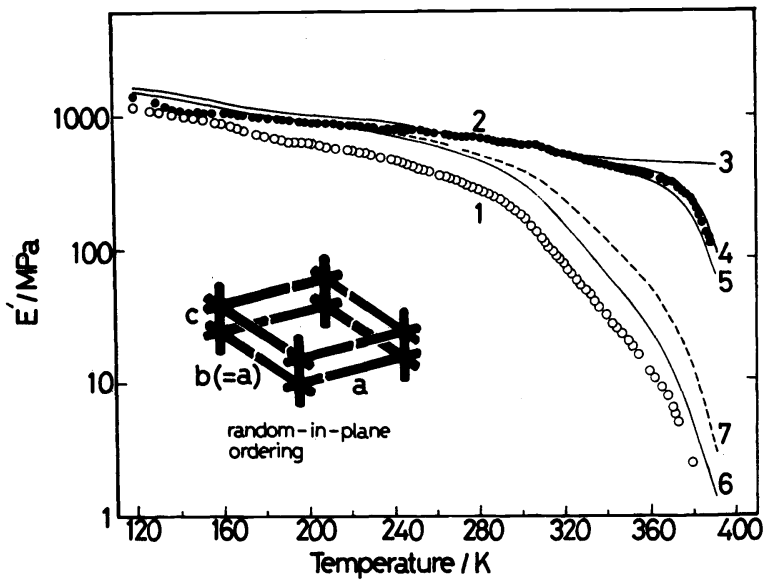

\begin{abstract}
Fig. 10. E' vs temperature for ionomer (curve 1) and the composite of the surface modified Kevlar filament/ionomer (curve 2). The solid lines represent the calculated curves based on the viscoelastic data of ionomer and volume fraction of filament $(2.2$ vol\%), assuming a quasi-3-dimensional lattice model in which $a=323 \mu \mathrm{m}$ and $c=a / 10=32 \mu \mathrm{m}$ with filament diameter of $12 \mu \mathrm{m}$. The $\phi$, of the fraction of interrupting the continuity of network strand is assumed to be 0 for curve $3,5 \times 10^{-5}$ for curve $4,1 \times 10^{-4}$ for curve 5 and 0.01 for curve 6 . Curve 7 is the one calculated by the modified Halpin-Tsai eqation (Ref. 11).
\end{abstract}

\title{
STRESS-STRAIN BEHAVIOR OF MOLECULAR COMPOSITES
}

The main interests in polymer composites are to clarify the difference from the macro-fiber reinforced composites. In the latter case, strong and hard filaments or fibers are embedded in a ductile matrix. Orientation of fibers is most desirable. When force is applied along the orientation direction of filaments, the shear stress is generated at the interface of filament and matrix, force is transferred to fiber to fiber when the adherence at the interface is strong enough. Crack propagation is interrupted by the presence of the interfacial boundaries. Repeated nucleation of crack is necessary for the crack to proceed. These requirments are in common for the macro-fiber reinforcements and the molecular composites employing rigid molecules. Usually shape of macro-fiber composites is constructed by assembling fibers and then, the matrix resin fills the space among the fibers. For this process, the fraction of fibers occupies the major space (e.g. 40\%). As seen in the examples of the molecular composites, the fraction of rigid molecules is only several percentages. Thus, the molecular composites are processable as raw polymeric materials, whereas the macro-fiber composites are rather rigid existence from the beginning. If three-dimensional reinforcement is desired, macro-fibers must be aligned along the three axes of Cartesian coordinates and then, the matrix resin is poured into the space. The molecular composites are composed of small volume fraction of in-situ crystallized 3-dimensional network of rigid microfibrils and the major fraction of matrix resin. Three-dimensional reinforcement has already been builtin in the composite. Application of force for orientation is of course possible, if unidirectional reinforcement is desired. The final problem is the comparison in the effect of reinforcement. In the following, the stress-strain behavior of the molecular composites will be surveyed. 


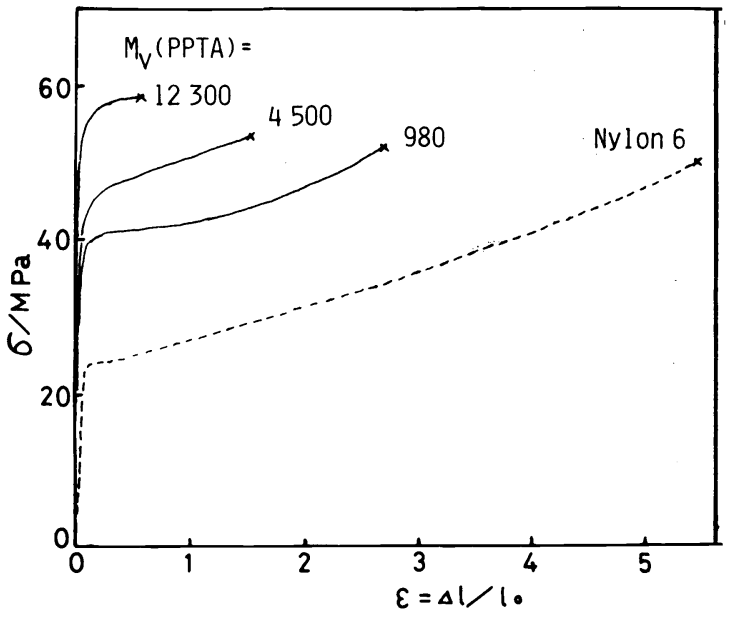

Fig. 11

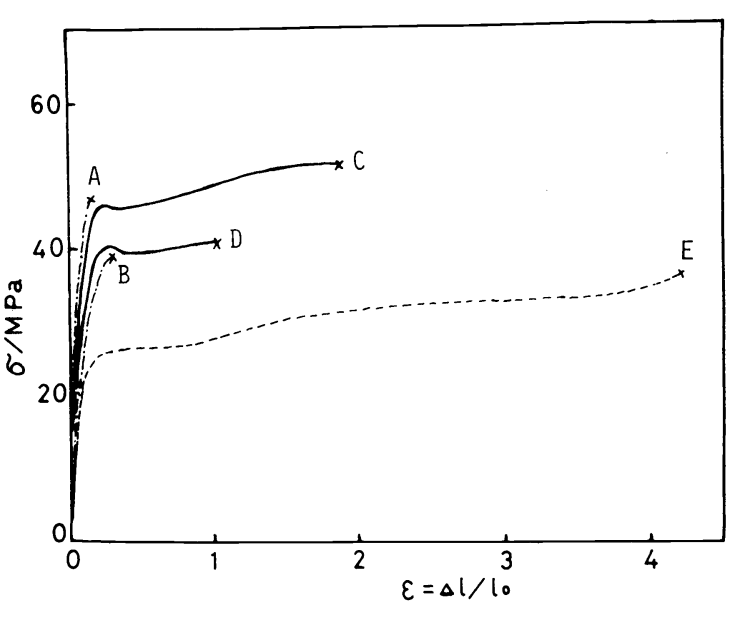

Fig. 12

Fig. 11. Nominal stress $(\sigma)$ vs strain $(\varepsilon)$ for (------) nylon 6 and $(\longrightarrow) 5 / 95$ composites of PPTA/nylon 6 with various molecular weights of PPTA.

Fig. 12. Stress-strain curves of (A, B) composites of PPTA and nylon 66 , (C, D) composites of the block copolymers of PPTA/nylon 66 and nylon 66 , and (E) pure nylon 66. The net blend ratio of PPTA/nylon $66=5 / 95$. The block composition is PPTA/nylon $66=28 / 72$ for curve $C$ and $37 / 63$ for curve D. The molecular weight of PPTA block is 4200. Molecular weights of PPTA for the samples of curves $A$ and $B$ are 4500 and 980 , respectively.

is statistically isotropic. With increasing molecular weight of PPTA, the yield stress ( $\left.\sigma_{y}\right)$ and the Young's modulus (E) increased markedly. Ultimate strength $\left(\sigma_{b}\right)$ did not vary so much, while the ultimate elongation $\left(\varepsilon_{\mathrm{b}}\right)$ decreased with increasing molecular weight. These results resemble those of macro-fiber reinforced plastics, in which as much as $40 \%$ of macro-fibers are employed. The explanation of these tendencies is that the PPTA molecules with high molecular weight form a more perfect and stronger microfibril than the low molecular weight ones, and the larger effect of reinforcement is expected in the former case. The hard and strong, but inextensible microfibrils of high molecular weight PPTA restrain the ductile deformation of polymer composite to give low ultimate elongation. The decrease of elongation with increasing molecular weight might be ascribed to the end of microfibril, which originates microcrack. In order to improve the extensibility of the composite while keeping the strength, dispersion of PPTA molecules into finer microfibrils might be desirable. For this purpose, the rigid block of PPTA or PBA was further bonded to the flexible chain blocks of nylon 6 or 66 . The preparation methods of block copolymers were given previously.

Figure 12 shows the stress-strain curves of the composites of multi-block copolymers and nylon 66 (solid lines) and the physical blends of the same components (chain lines). The net weight ratio of PPTA/nylon 66 was adjusted to 5/95 for both cases. The curve of nylon 66 (broken line) is shown for comparison. The molecular weights of PPTA in the samples of physical blends employed in the sress-strain experiments of A and B were 4500 and 980 , respectively. The extensibility of nylon 66 is entirely lost in the physical blends for $A$ and B. On the other hand, the improvement of ultimate elongation for the composites of block copolymers of PPTA/nylon is remarkable as seen in curves C and D. The flexible block molecules connected to the rigid PPTA block will be effective in forming lamellar crystals with the matrix flexible molecules. After yielding of the specimen, the unfolding of nylon 66 molecules continues with the help of the rigid blocks playing a role of tie molecules. With this mechanism, the improvement of ultimate elongation was achieved. The sample of curve $C$ is a multi-block copolymer of PPTA/nylon $66=28 / 72$, while the sample of curve D is $37 / 63$. The block length of sample $C$ is longer than that of sample $D$, with the same PPTA length of $\mathrm{M}_{\mathrm{V}}=4200$. The efficiency for reinforcement is higher in the block copolymer with longer flexible block. Similar results were obtained for the composites of triblock of PPTA/nylon 6 and multi-block of PBA/nylon 6 (Ref. 1).

PPTA microfibrils and PVC

Figure 13 shows the comparison of the stress-strain curves at room temperature. A remarkable extensibility in as-cast PPTA microfibril/PVC film (curve 1) seems to be caused by the existence of many voids, with which PVC matrix became plastic by generating many crazes. At that time, strong PPTA microfibrils or their aggregates (fibril) support the generated stress. But, referring to Fig. 4, the fractured surface inspected by SEM reveals that the fibrils are 
pulled out from the matrix of PVC after occurrence of p.lastc deformation. No increase of yield stress was observed for the composites compared with PVC sheet. Thus, it may be concluded that the adhesion of PVC to the surface of PPTA microfibrils is not so strong as to result in the fracture of PPTA fibrils, but to some extent, the frictional resistance at the interface is enough to dissipate strain energy to result in ductile deformation of PVC matrix. The strong interaction between PVC and benzanilide as a model substance of PPTA was proved by the shift of characteristic bands associated with hydrogen bond. With increasing the annealing temperature, the adherence is improved and yield stress is increased. But it is not enough to bring fracture of PPTA fibril and remains only to make the strain energy for pulling-out of fibrils increase, or in other words, the composite is more toughened.

Once temperature is raised to $80^{\circ} \mathrm{C}\left(5^{\circ}\right.$ higher than $\left.\mathrm{T}_{\mathrm{g}}\right)$, adherence at the interface is 1 argely improved and typical effects of reinforcements can be seen. Modulus and strength are increased as in the case of macro-fiber reinforcement. At $80^{\circ} \mathrm{C}$, 7 wt\% of PPTA provides the composite with yield stress of $13 \mathrm{MPa}$, while PVC is in a rubbery state.

Cured composites of PPTA and NBR

Figure 14(a) shows the stress-strain curves for the PPTA-loaded cured NBR, black stock and gum stock. NBR raw rubber contains $40 \mathrm{wt} \%$ comonomer fraction of acrylonitrile. Effect of molecular weight is clear. The samples of PPTA with high molecular weight of 21900 and 25100

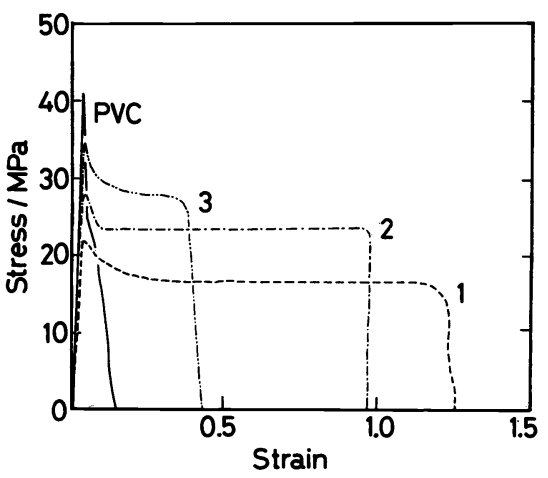

Fig. 13

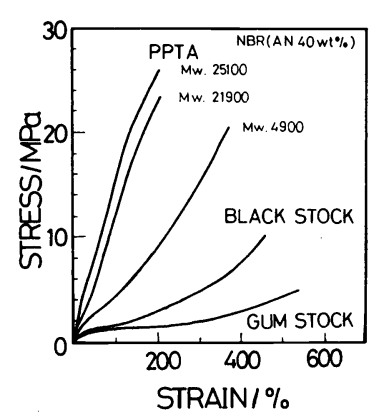

(a)

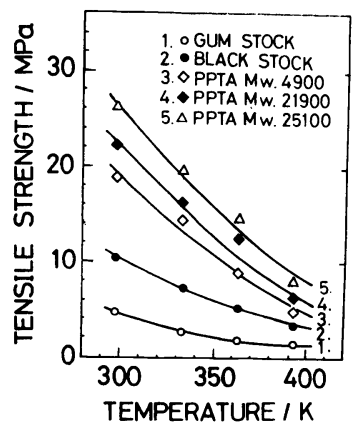

(b)

Fig. 14

Fig. 13. Stress-strain curves for the composites of PVC and PPTA micro- or macro-fibrils with 2.4 wt\% of PPTA. Curve 1 : as-cast composite sheet (turbid), curve 2: heat-pressed sheet at $160^{\circ} \mathrm{C}$ (tranparent), curve 3: the same as in curve 2 with longer press time.

Fig. 14 (a) Stress-strain curves of NBR gum stock, black stock and the polymer composites of PPTA with various molecular weights as indicated in the figure. PPTA in the composites and carbon black are filled with 6.4 vol\%. (b) Temperature dependence of tensile strength for the same samples as in (a).

are strong and hard compared with the sample of molecular weight of 4900 . The curve of black stock is obtained for the same content of carbon black as the PPTA content of 6.4 vol\% of the composites. Microfibrillar PPTA is very effective for reinforcing rubber compared with carbon black (here ISAF is used). It should be noticed that the modulus of the composites is superior to the standard vulcanizate of NBR with highly loaded carbon black ( $40 \mathrm{phr})$. Figure $14(\mathrm{~b})$ shows the temperature dependence of tensile strength for the same samples as in Fig. 14(a). With increasing temperature, the strength decreases. However, the order of samples does not vary. Here again, the data are superior to those of NBR with highly loaded carbon black. In the case of elastomers, the matrix is in a rubbery state and the rigid microfibril as reinforcement is very effective due to the great difference in their moduli. Quasi-3-dimensional network acts strong resistance to make large deformation, in addition to the effect of strong interfacial interaction between PPTA and NBR matrix.

Effect of carbon black is caused by the particulate form and its structure, in addition to the strong interaction of the surface of carbon black with rubber matrix. But, in this case, microfibrillar PPTA has a characteristic which can not be realized by carbon black.

Finally it must be noticed that the high tear strength is the most successful character of the polymer composites of PPTA/NBR. Tear strength evaluated by using trouser-shape specimen (JIS K 630L, B) reaches $70.3 \mathrm{kN} / \mathrm{m}$ for the composite including 6.4 vol\% PPTA, whereas that of black stock with the same volume fraction of carbon is on $1 \mathrm{y} 15.6 \mathrm{kN} / \mathrm{m}$. Such remarkable effect of microfibrillar PPTA on high tear strength might be ascribed to its resistance to be pulled out from the matrix.

Surface-modified Kevlar fiber-reinforced ionomer

Figure 15 shows the stress-strain curves for (a) ionomer, (b) the composite of PPTA fibers and ionomer, (c) the composite of PPTA fibers with the surface modified by carboxymethyl group and ionomer, (d) the composite of PPTA fibers with the surface modified by acrylonitrile 
and ionomer, and (e) the composite of carbon fibers and ionomer at room temperature. The volume ratio of reinforcement/ionomer is $2.2 / 97.8$ for all cases. The effects of type of functional group are remarkable. The composites of ionomer and unreacted PPTA fibers or the surface-modified PPTA fibers by carboxymethyl groups are remarkably improved in their yield stresses and Young's moduli, even by as small as 2.2 vol\% of PPTA fibers, compared with ionomer only. Especially, in the case of the latter, the yield stress increased from 10.0 to 33. $\mathrm{MPa}$ and the Young's modulus increased from 152 to $783 \mathrm{MPa}$. The ultimate strength increased a little from 19.7 to $27.1 \mathrm{MPa}$, while the uitimate elongation decreased from 705 to $27 \%$. As for the PPTA-g-acrylonitrile, the interfacial interaction is weak, but the acrylonitrile branch is effective to increase the extensibility, perhaps owing to the anchoring of the branch into the matrix. Carbon fiber has essentially higher modulus than Kevlar fiber. According to the results shown in Fig. 15, the surface interaction is a more important factor than the modulus of fiber itself. The explanation of unusually high efficiency of the surface modified PPTA filaments might be ascribed to the capability of this system for forming quasi3-dimensional lattice with chopped strands of Kevlar filaments. Such a conclusion is derived from the behavior of the other molecular composites. However, at present there is no definitive evidence from the structural characterization. Micro-domains of ca. $2 \mathrm{~nm}$ in diameter in ionized state dispersed in ionomer matrix are considered as one possible factor.

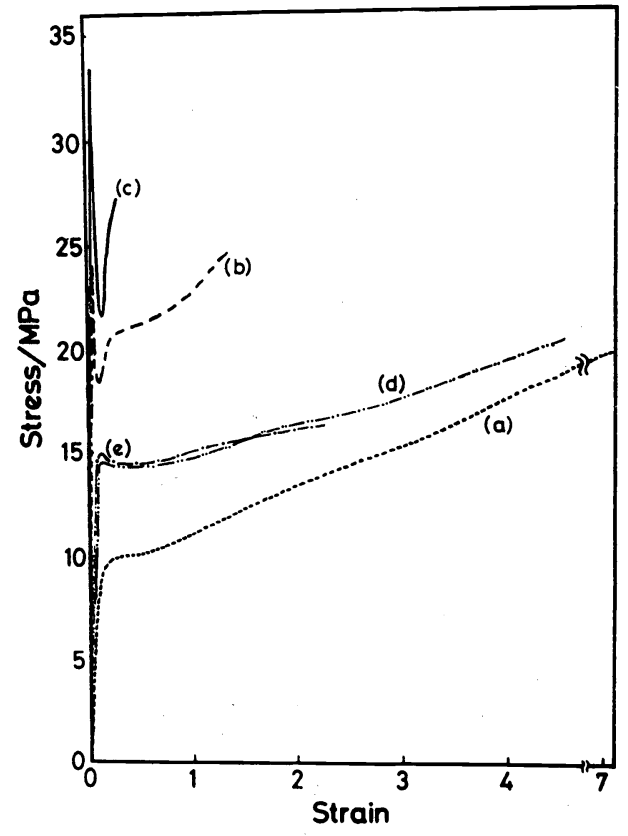

Fig. 15. Stress-strain curves for (a) ionomer, (b) the composite of PPTA fibers and ionomer, (c) the composite of PPTA fibers with the surface-modified by carboxymethyl group and ionomer, (d) the composite of PPTA fibers with the surface-modified by acrylonitrile and ionomer, and (e) the composite of carbon fibers and ionomer at room temperature. The volume ratio of reinforcement/ionomer is $2.2 / 97.8$ for all cases.

To form a quasi-3-dimensional network with very hard and strong rigid strands in the matrix of ductile polymers is sole possible conclusion to be quoted on the reinforcing factor based on various examples cited above. Strong adhesion at the interface of both materials is necessary condition for achieving successful composite. Very small amount of reinforcement and realization of 3 -dimensional reinforcement keeping the processability are also cited as the advantages of our systems.

\section{REFERENCES}

1. M. Takayanagi, T. Ogata, M. Morikawa and T. Kai, J. Macromol. Sci.-Phys. B17, 591-615 (1980).

2. M. Takayanagi and T. Katayose, J. Polym. Sci.: Polym. Chem. Ed. 19, 1133-1145 (1981).

3. M. Takayanagi, Preprints of 2nd Japan-Korea Joint Symposium on Polymer Sci. and Technology, Oct. 20-22, 1980, Kyoto, 61-66 (1980).

4. W. J. Jackson, Jr. and H. F. Kuhfas, J. Polym. Sci.: Polym. Chem. Ed. 14, 2043-2058 (1976).

5. E. I. du Pont de Nemours, U.S. Patent 3817941 (1974).

6. N. Yamazaki, M. Matsumoto and F. Higashi, J. Polym. Sci.: Polym. Chem. Ed. 13, 1373 (1975).

7. T. Kai, T. Kajiyama and M. Takayanagi, Rep. Progr. Polym. Phys. Japan, 24, 163-166 (1981).

8. T. Oono, F. Kumamaru, T. Kajiyama and M. Takayanagi, Rep. Progr. Polym. Phys. Japan, 24, 193-196 (1981).

9. M. Takayanagi and T. Katayose, submitted to J. Appl. Polym. Sci. (1982).

10. K. Haraguchi, T. Kajiyama and M. Takayanagi, SEN-I GAKKAISHI (J. Soc. Fiber Sci. Tech. Japan), 33, 535-540 (1977).

11. L. E. Nielsen, Mechanical properties of polymers and composites, Mercel Dekker, New York (1975). 